\title{
Design analysis and performance evaluation of the WDM integration with CO-OFDM system for radio over fiber system
}

\author{
Adnan Hussein Ali ${ }^{1}$, Hayder Jasim Alhamdane ${ }^{2}$, Begared Salih Hassen ${ }^{3}$ \\ ${ }^{1}$ Middle Technical University, Technical Instructors Training Institute, Iraq \\ ${ }^{2}$ Middle Technical University, Institute of Technical managrment, Baghdad, Iraq \\ ${ }^{3}$ Technical Electronics Department Institute of Technology/ Baghdad, Middle Technical University, Baghdad, Iraq
}

\begin{tabular}{l} 
Article Info \\
\hline Article history: \\
Received Oct 1, 2018 \\
Revised Dec 10, 2018 \\
Accepted Jan 25, 2019 \\
\hline
\end{tabular}

\section{Keywords:}

BER

Radio over fiber

Wavelength division

multiplexing

WDM-CO-FDM RoF

Q- factor

\begin{abstract}
The optical communication and wireless networks can be integrated to increase the capacity and mobility with decrease the costs in the access networks. Optical communication is considered a Fiber optic communication that can be used for wired and wireless communication. The problems of Fiber optic communication have a solution represented by Radio over fiber (RoF) which can control many base stations (BSs) that connect to a central station (CS) with an optical fiber. The Coherent optical OFDM (CO-OFDM) have concerned a great interest due to the high spectral efficiency and strength to the fiber dispersion, also it can be considered as a promising candidate when used in long haul optical fiber transmission systems. The integration of the wavelength division multiplexing system (WDM) with (CO-OFDM) system for increasing the system performance as well as achieving high data rates. The simulation of WDM-ROF and (WDM-COOFDM) with an optisystem simulator to analyze the RF spectrum, spectrum signal visualizers, and constellation diagrams. The proposed of integration of WDM and RoF-OFDM system is to achieve $100 \mathrm{~km}$ fiber length using 4QAM sequence bit, and the received power with electrical and optical amplifications by using $10 \mathrm{Gbps}$ data signal.
\end{abstract}

Copyright $(2019$ Institute of Advanced Engineering and Science. All rights reserved.

\section{Corresponding Author:}

Adnan Hussein Ali,

Middle Technical University,

Technical Instructors Training Institute, Baghdad, Iraq.

Email: aaddnnaann63@gmail.com

\section{INTRODUCTION}

The internet traffic increasing that includes voice and video in addition to data services, has directed to increased demand in high data rates and bandwidth. The quick Internet traffic evolution and the emerging applications are considering the significant driver for high-capacity and cost-effective optical fiber transmission technologies. The Fiber-optic communication is a technique of transmitting information between one place and another through sending a light in an optical fiber [1]. Fiber-optic communication systems have primarily been installed in long-distance applications, where they can be used to their full transmission capacity, compensating the increased cost. Optical transmission systems are popular for maintaining low power for short to medium range wireless communications. But for other applications where transmission distance is large, high power is required. In an optical fiber, various nonlinear effects begin to appear as the optical power level increases [2].

Radio over fiber (RoF) considers as an analog optical link transmitted modulating RF signals. It supports to transmit the RF signal downlink and uplink, bidirectional from the central station (CS) to the base station (BS). These RoF systems have not only the high speed, large capacity, and low loss characteristics of the optical fiber communication, but also the flexibility of the mobile communication meeting the development trend of future 5G mobile communication and the demand for gigabit magnitude services [3]. 
In fiber-optic communications, the technology of wavelength-division multiplexing (WDM) in which multiplexes a number of optical carrier signals with several wavelengths onto a single optical fiber by using different wavelengths of laser light to carry different signals, and it can achieve a greater capacity, higher data rate and better flexibility at relatively low cost and system can be easily upgraded and also helps bi-directional transmission over a single fiber length for transmitter and receiver [4].

Orthogonal frequency-division multiplexing (OFDM) can be labeled as multicarrier modulation that a data information is carried with various lower rate subcarriers. Two main advantages of OFDM can be achieved, which are its robustness against channel dispersion as well as its ease of channel and phase estimation in a time-varying environment [5]. The wavelength range of WDM bands is an approximation value and has not yet been standardized. Considering O-band which is $1260 \mathrm{~nm}$ to $1360 \mathrm{~nm}$ range is equivalent to $14 \mathrm{THz}$ bandwidth. Meanwhile, combination of S-band and C-band with a range of $1460 \mathrm{NM}$ to $1625 \mathrm{NM}$ provides another $15 \mathrm{THz}$ bandwidth. The total available bandwidth per fiber optic in O- band, S-band and C-band only is around $30 \mathrm{THz}$ of the low-loss regions of a standard G.652 single-mode fiber [6].

This paper focuses on the transmission and reception of data streams over a range of optical fiber by using an integration of WDM with CO-OFDM scheme. The purpose is analyzing the WDM- RoF network execution, and studying the OFDM and CO-OFDM integration. Then evaluate CO-OFDM with WDM for long-haul by studying the constellation diagram of the system with different modulation schemes. The simulation of the proposed systems by using OptiSystem simulation tool. Finally, analyze the effect of the transmission distance on the CO-OFDM and WDM.

\section{METHODS AND MATERIALS}

\subsection{Radio Over Fiber (RoF)}

In particular, the RoF system, which is inherently compatible with wavelength-division multiplexing (WDM) technology, can efficiently support a large number of base stations (BSs) required to cover wireless service areas in Pico- or Femto- cell configuration [7]. The demand to have broadband capacity as a wireless has put pressure on wireless communication systems to increase both their transmission capacity, as well as their coverage. The RoF systems have not only the large capacity, high speed, low loss characteristics of the optical fiber communication, but also the flexibility of the mobile communication meeting the development trend of future 5G mobile communication and the demand for gigabit magnitude services [8].

RoF is a very appropriate system as it is a lesser amount of power consumption and costing. That's due to RoF system lets the electrical signal can be modulate the optical source and then the optical signal will propagate through the optical fiber to the remote station. If the RF signal is modulated directly to the optical link, then a power consumption will be dropped while the side of the antenna has carriers with high radio frequency. The cost reduction in RoF technology can be demonstrated in two stations, the first is central station (CS), that's provides resources which may be shared by a set of base stations (BS), while the second in BS that can only execute simple tasks. Also, the size of BS is small and consume less cost.

High frequencies in the range of $\mathrm{GHz}$ have more bandwidth, hence their data carrying capacity or more, but they encounter more losses when they are transmitted wirelessly hence they cannot be transmitted long distances whereas the low frequency signals encounters less losses hence they can travel long distances. At high frequency associated losses are high. Hence, there is a need for a waveguide to carry these waves.

Basically, in RoF, the CS is connected with many BS with optical fiber. The function of BS only as an optical signal converter into a wireless signal and vice versa, whereas at CS, modulation, demodulation, routing and coding are all process that executed. The RoF system distributes the RF signal by utilizing high linear optic link between CS and BSs.

In order to further increase the bandwidth and the capacity of a wireless system, the WDM technique has been employed in ROF systems (WDM-RoF). Wireless network based on radio over-fiber technologies is a very beneficial solution. It acts as a back-end technology for the transportation of microwave and millimeter waves [9]. Integration of WDM with RoF leads to significant increase in the coverage area and the overall capacity of the current optical network, also to exploit the benefits of high data rate and increase mobility. A WDM-RoF system is proposed for signal demodulation. This has eradicated the need to design and install two separate networks.

\subsection{Wavelength Division Multiplexing (WDM)}

The Wavelength Division Multiplexing can be seen as an important feature in the expansion of the optical communications. The ability of WDM has provided more flexibility to the system and simplified the network design. It helps the enhancement of the system capacity by sending multiple wavelengths over a single fiber. A considerable increase in the data rate can be provided by WDM systems that are passed over a single fiber via multiple wavelengths using, where a separate channel is carried at each wavelength. 
In WDM, the optical spectrum is divided into smaller channels; each can be used for transmitting and receiving data simultaneously [10].

For WDM system, each wavelength can be preserved as discrete channel and different data is carried. WDM multiplexer can be considered passive devices which light signals are combined with different wavelengths, at a single fiber. The using of WDM for the RoF signals distribution has gained importance in recent times. The WDM is enabling the effective exploitation for the fiber bandwidth. The optical filters selection and wavelength stability limitations of semiconductor lasers, as well as the minimum channel spacing for the commercial WDM are approximately $50 \mathrm{GHz}$. This channel spacing can be reduced to 50 $\mathrm{GHz}$ or equal to $25 \mathrm{GHz}$ with a possibility for using hundreds of channels [11].

The optical WDM network is illustrated in Figure 1, which a substitution of the wavelength of frequency and a separated wavelength $\lambda \mathrm{i}$ is transmitted by each transmitter to different receivers. The WDM has the ability to transmit different frequencies without any interference in radio broadcasting. Two types of WDM systems include, Dense Wavelength Division Multiplexing (DWDM) in addition to Coarse Wavelength Division Multiplexing (CWDM).

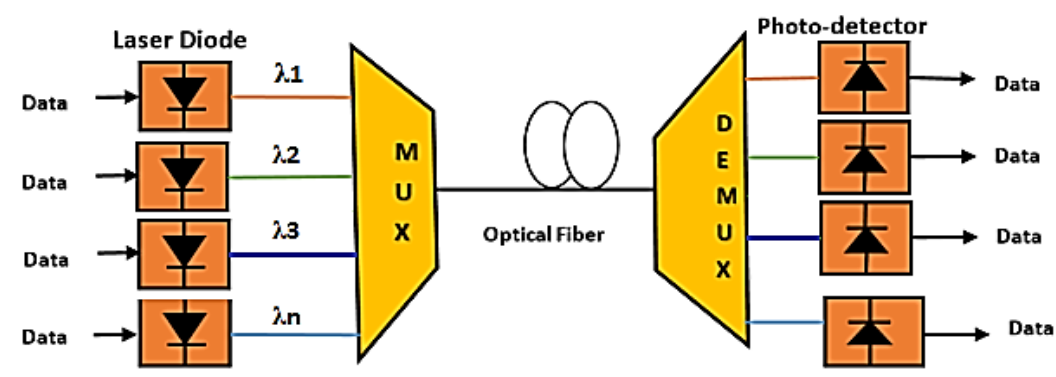

Figure 1. WDM component diagram

In WDM technique, $\mathrm{N}$ optical wavelength numbers can be used as a data carrier, and these wavelengths are combined with an optical fiber utilizing WDM multiplexer, while at the receiver, the separation of these wavelengths as individual channels at WDM demultiplexer. Each optical wavelength is different and typical; channel spacing will determine the gap between those wavelengths, so that overlapping optical carrier can be avoided [12].

The Subcarrier Multiplexing (SCM) can be considered as a complement of multiplexing techniques which can be utilized in optical system for increasing the efficiency of the bandwidth application. In the RoF system with SCM technique, the multiple RF signals are multiplexing in frequency domain and then transmitted at a single wavelength. SCM can be combined with wavelength division multiplexing (WDM) to provide a more flexible technique for optical transmission at high speed with high optical bandwidth efficiency in addition to high dispersion tolerance. This combination is more sensitive to the noise effects, but there is a disadvantage, which represent, by the limitation of the maximum subcarrier frequencies and data rates.

\subsection{Integration of WDM with CO-OFDM}

OFDM can be seen as an improved form of Frequency Division Multiplexing (FDM), where in this technique, many information for several users will be transmitted simultaneously over different frequency carriers. This can be shown in Figure 2 wherein the transmitter section, each subcarrier is fixed with a wide guard band when it is modulated by means of the user's data for preventing it from interfering with the adjacent subcarriers. So that, this guard band will decrease the system spectral efficiency. At the receiver, the received signals will be demodulated with oscillator banks [13].

\section{METHODOLOGY OF THE PROPOSED SYSTEM}

\subsection{Description of WDM-RoF System}

The suggested system consists of four RF signals transmitted over single mode optical fiber at 60 $\mathrm{km}$ and received by 4 remote stations. RoF network with Optical communication can be simulated and implemented by using Optisystem, which is a powerful and an inventive software design tool and enables users for designing, testing and simulating virtually each optical link kind positioned in transmission layer of the broad spectrum of the optical networks [14]. 
The block diagram of the proposed WDM-RoF system can be shown in Figure 3. The transmitter comprises of channels, and each channel has its own operating wavelength, channel 1 is $1552.50 \mathrm{~nm}$ with a linewidth of $10 \mathrm{MHz}$, and so on. As a result, a wavelength spacing equal to $0.4 \mathrm{~nm}$ between subsequent channels is maintained for low and smooth transmission. As the separation is narrow, helps to support big client numbers and to accomplish a high rate. Each channel has an input power to be changed from $-15 \mathrm{dBm}$ to $15 \mathrm{dBm}$ with a step of $5 \mathrm{dBm}$. The WDM multiplexer that have a bandwidth of $10 \mathrm{GHz}$ can combine the signal which comes from the input channel then transmits it over the optical fiber. The optical fiber length is $60 \mathrm{~km}$. In optical communication links, EDFAs are used for enhancing the signal power to the all channels simultaneously.

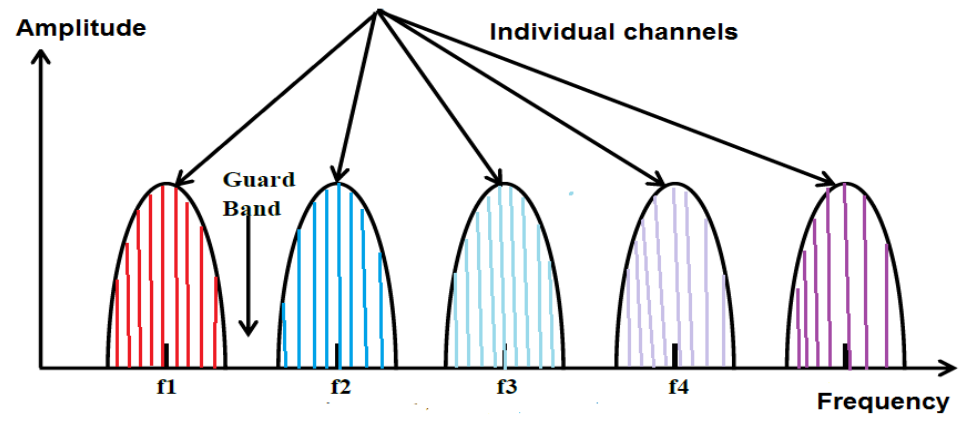

Figure 2. OFDM subcarrier transmitter

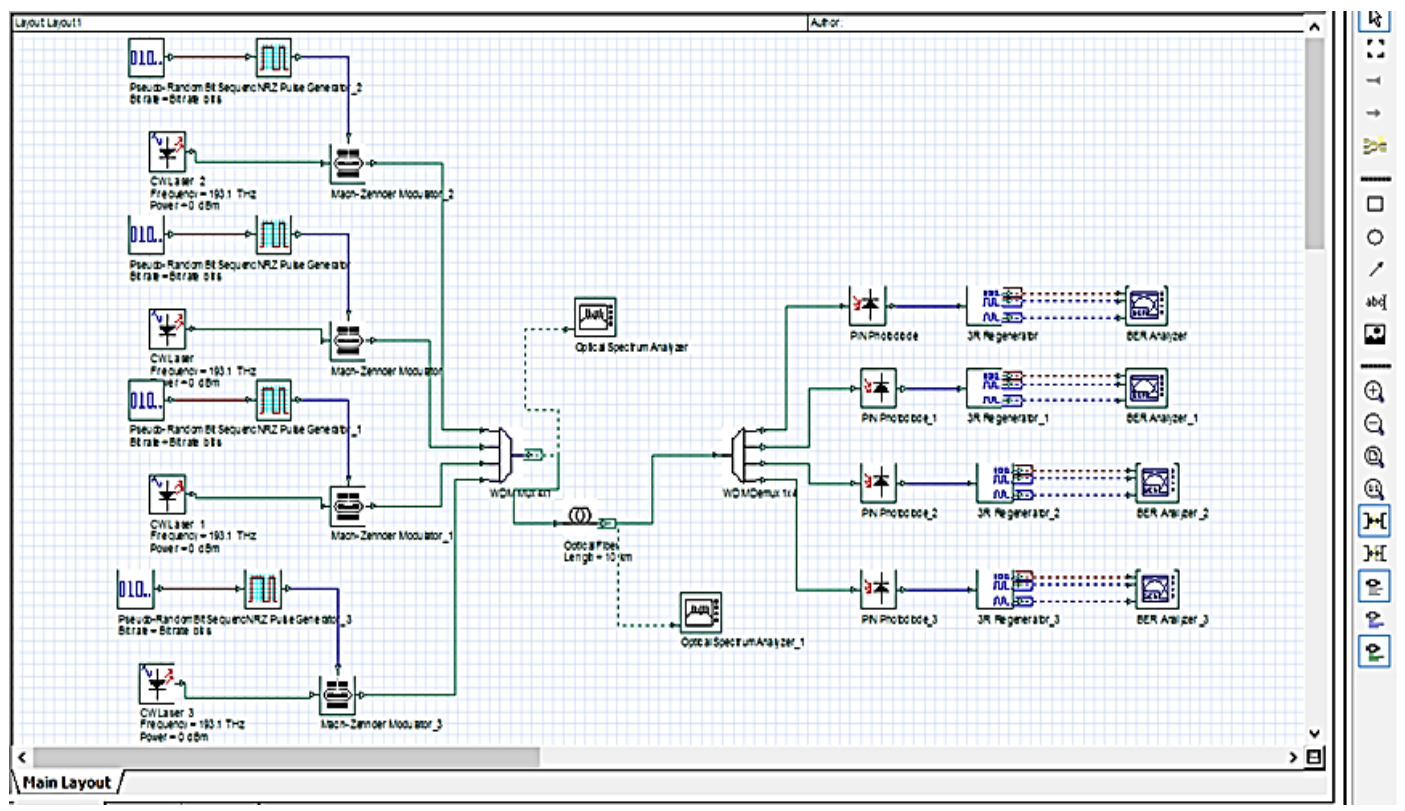

Figure 3. The proposed WDM-RoF system

The proposed WDM multiplexer of $10 \mathrm{GHz}$ bandwidth has a four signals combination, each from one input channel and then a transmission of them over the optical fiber link which operates in the wavelength window of $1550 \mathrm{~nm}$ and requests for dispersion management.

At the transmitter, the binary data supplied by PRBS (Pseudo- Random Bit Sequence) are converted into Non Return to Zero (NRZ) electrical pulses to generate a baseband signal. The information rate for the reenactment of both the downlink and uplink signal is set to 1 Gbps. The carrier frequency of the electrical amplitude modulator is $1.7 \mathrm{GHz}$ can be used to convert the frequency of baseband signal to the RF clock frequency.

The RF signal is applied to $90^{\circ}$ hybrid coupler after SCM carrier generator as shown in Figure 4. Further, the outputs of hybrid coupler are connected to dual port, dual drive, Lithium Niobate Mach- Zender 
modulator, LiNbO3 MZM. The MZM is used for the transmission of microwave signal into an optical signal. The optical signal obtained at the output port of MZM is further transmitted over the optical fiber link.

Then, the optical modulated signal will be passing through SMF channel. This optical link model utilizes an SMF which confirm a good quality level through the transport of signals. The attenuation factor value can be chosen with $0.2 \mathrm{~dB} / \mathrm{km}$.

At the receiver, the optical modulated signal through the fiber is directly detected on the PIN (positive intrinsic negative) photodiode with a suitable sensitivity and the photocurrent represents a replica of the modulated RF signal applied externally by means of MZM modulator at the transmitter. This PIN photo diode converts the optical signal to electrical signal, then amplified through an electrical amplifier for making up the power loss owing to the attenuation in fiber. A gain of $20 \mathrm{~dB}$ confirms that all distorted signals which are attenuated through transmission by fiber channel may be recovered at the receiver.

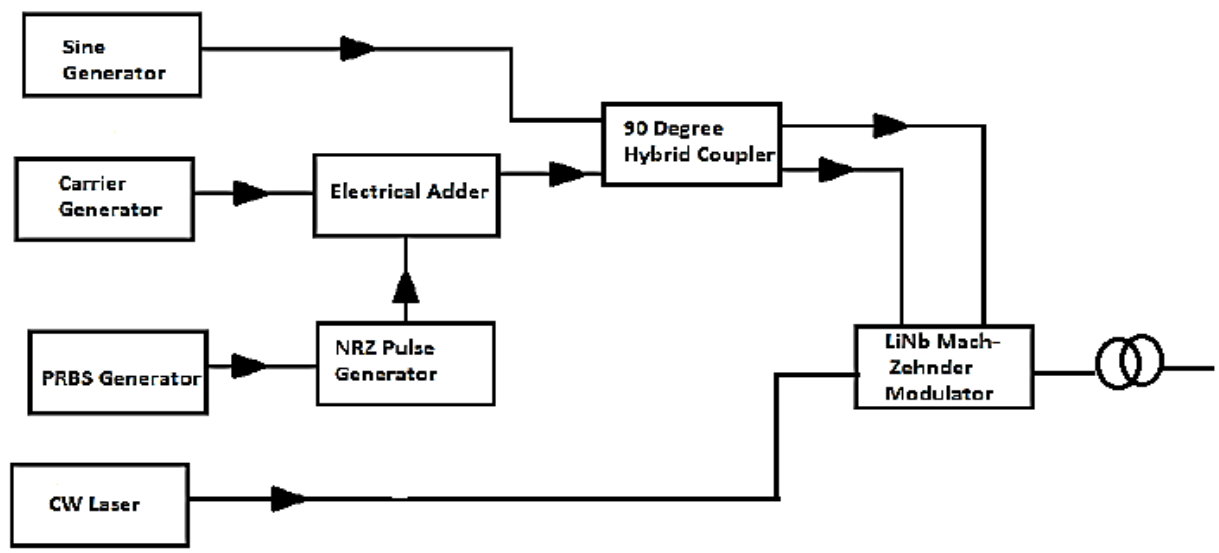

Figure 4. WDM transmitter

\subsection{Integration of WDM with CO-OFDM Schems}

WDM can be used to provide more flexibility for the system as well as to simplify the network design and to enhance the network capacity by utilizing multiple wavelengths over a single fiber which gives a result of the data rate will be increased. The design of WDM-CO-OFDM system can be shown in Figure 5 with a $100 \mathrm{~km}$ SMF length. The CO-OFDM transmitter is constructed with a Pseudo Random Binary Sequence (PRBS) for generating a bit sequence which will approximate the random data characteristics. It is also built with a 4-QAM that has 2 bits per symbol encoder. The OFDM modulated producing 512 subcarriers at the position of 4-QAM and at the 1024 FFT points. The resulting signal with in-phase (I) and quadrature $(\mathrm{Q})$ of the OFDM modulator will be transmitted to the I/Q optical modulator. This optical modulator involves two LiNb (lithium Niobate) MZM (Mach-Zehnder modulators) that the OFDM modulator electrical signal will be modulated to the optical carrier. This optical carrier which has laser wavelengths centered at $193.1 \mathrm{THz}$ and started from $193.05 \mathrm{THz}$ to $193.2 \mathrm{THz}$.

The WDM system consists of four channels to support the four OFDM bands with channel spacing of $50 \mathrm{GHz}$. Each OFDM signal has a $12 \mathrm{Gbps}$ bit rate which will provide an overall data rate of $48 \mathrm{Gbps}$. The resulting signals from the OFDM transmitters are launched into the WDM MUX and filtered by a Gaussian optical filter. The four different wavelengths are merged to produce one signal to be launched on a single fiber. The resulting optical signal of the WDM MUX is then transmitted through the SMF. The SMF attenuation is $(0.2 \mathrm{~dB} / \mathrm{km})$ and the dispersion is $(16 \mathrm{ps} / \mathrm{nm} / \mathrm{km})$ for $100 \mathrm{~km}$. SMF will produce a dispersion of $16 \times 100=1600 \mathrm{ps} / \mathrm{nm}$. An Erbium Doped Fiber Amplifier (EDFA) is used with (20 dB) gain to amplify the signal and to compensate for the loss.

The Design of the receiver system takes a consideration for long variety of fiber optic when using a loop control. The Optical Filter Frequency is used to predict the losses that caused by dispersion and scattering when it pass on fiber of $100 \mathrm{~km}$ Length. At the start of the receiver, there is an optical detector which consists of photodetector PIN having an external source that it produces by a local oscillator. The conversion of optical signal into an electrical current at PIN photodiode component is based on the photodiode responsivity. The incoming optical signal with a noise bins will be filtered to decrease the sample number in the electrical signal. The center frequency is produced from new sample rate. The Schematic of RF transmitter and optical receiver is shown in Figure 6(a) and (b). 


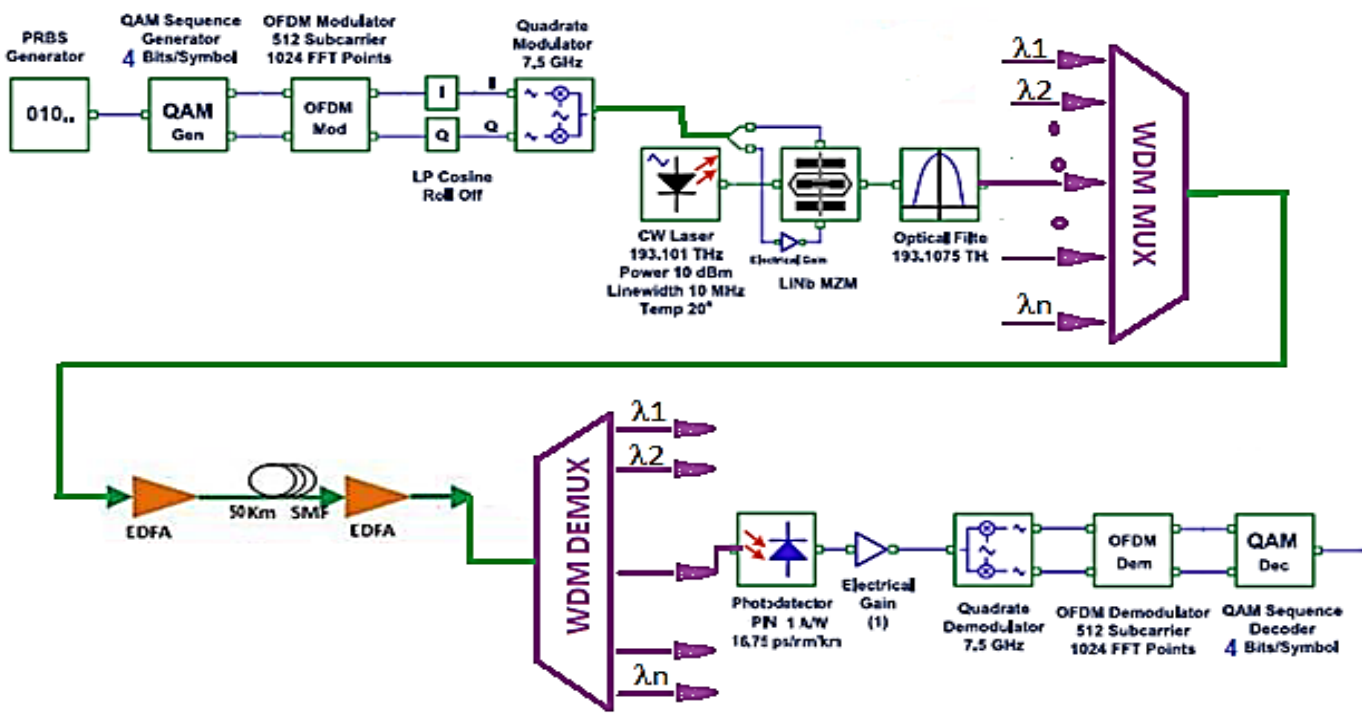

Figure 5. WDM-CO-OFDM system

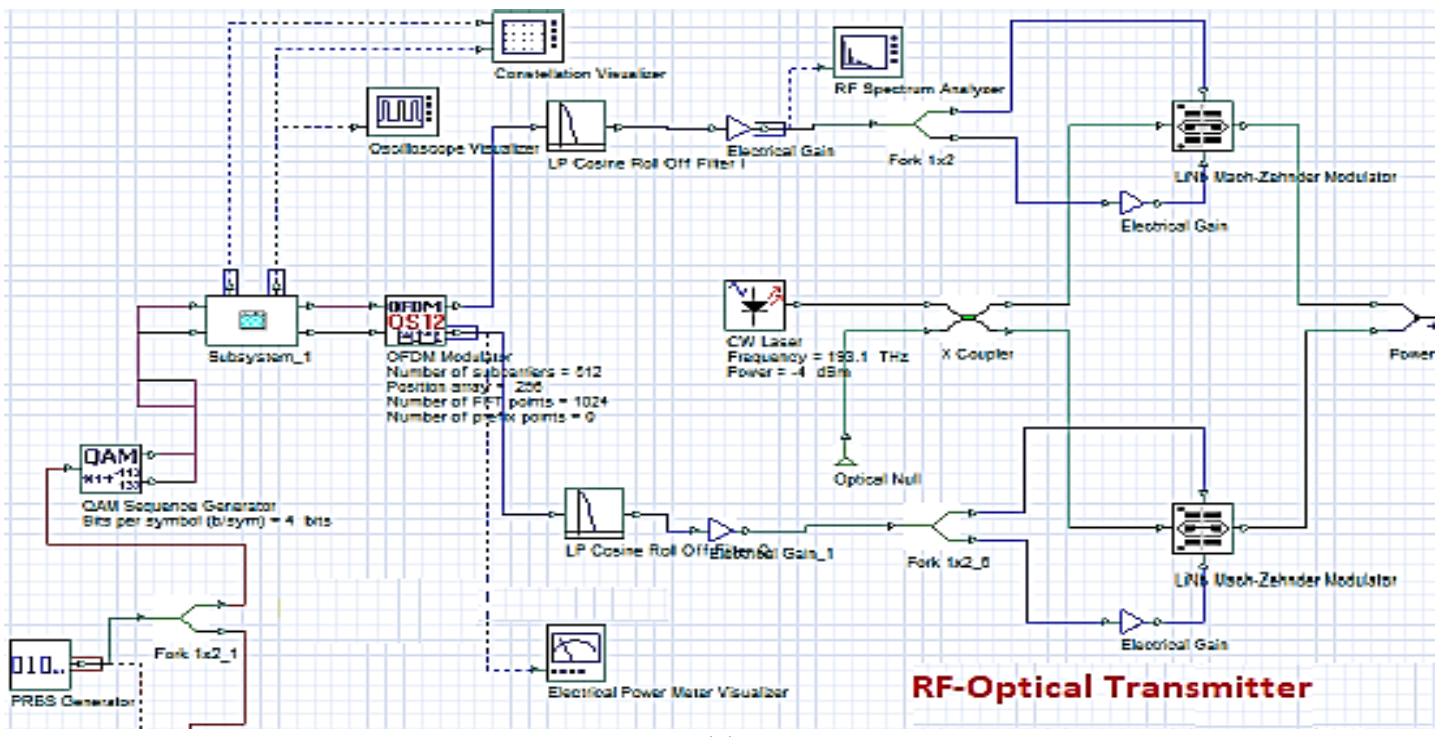

(a)

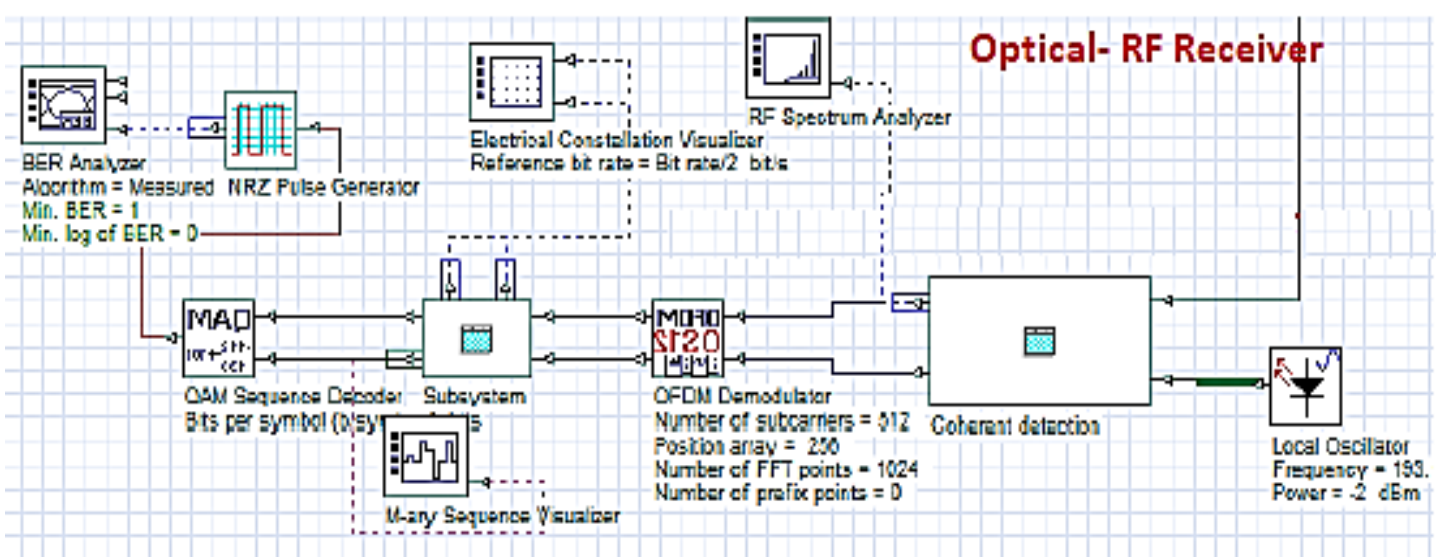

(b)

Figure 6. Schematic of RF transmitter (a) and optical receiver (b) 
Then an inverse part of the RF OFDM transmitter exists, which involves the OFDM demodulator plus the QAM decoder. The result of the received O/E conversion gives a carrier frequency signal which will be demultiplexing to get an output signal.

The single parameter optimization (SPO) can benefit a lot in the simulation. It helps to optimize parameters so we could set a target for the simulation's result. With optimization tools, the software can optimize the fiber length of the EDFA so that a maximum gain could be obtained. It can also calculate the attenuation or the gain in order to get a desired Q factor and minimize the BER by optimizing the fiber length of the system.

\section{RESULTS AND DISCUSSION}

The proposed WDM-RoF network is positively simulated and analyzed using Optisystem 13, a commercial optical system simulator. Figure 7 shows the optical spectrum after getting multiplexed by WDM multiplexer. The spectrum is observed by using an optical spectrum analyzer at the output of WDM multiplexer. After all components had been connected and SPO had been inserted to the topology, the simulation is run to see the result. After the run button is clicked, it will calculate all the calculation of the system. To measure the BER and the Q factor, just simply double click at the BER analyzer component. It will give an eye diagram showing eye opening, BER value and $\mathrm{Q}$ factor value.

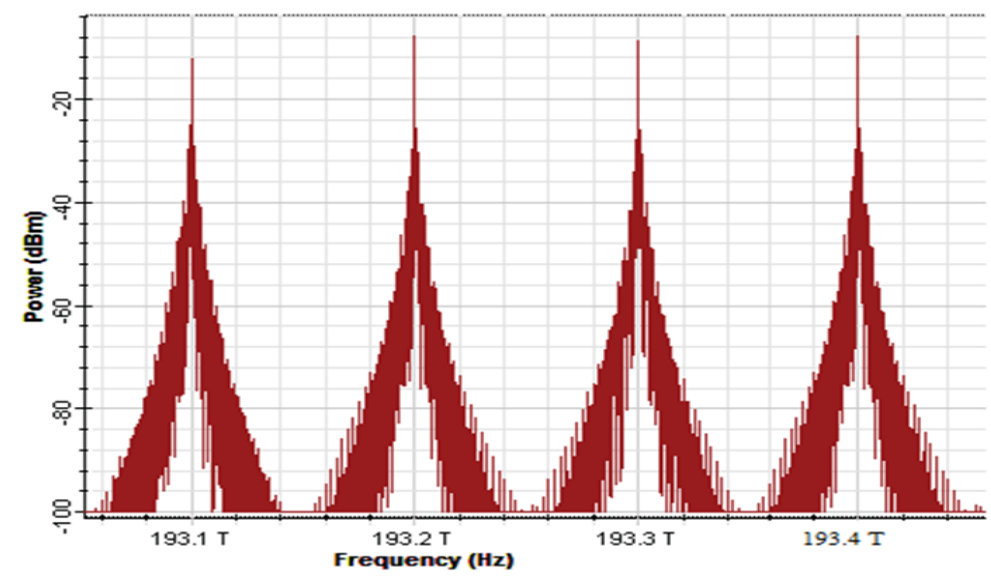

Figure 7. Optical spectrum multiplexed by WDM

After the simulation is done, the performance of the receiver is analyzed by referring the BER analyzer and the eye diagram at the receivers. The results can be seen in Figure 8. For receiver 1 with $10 \mathrm{~km}$ fiber length, the BER analyzer shows that the minimum BER for the received signal is $1.1009 \times 10^{-10}$. The $\mathrm{Q}$ factor for this receiver is 6.3046. This shows that this receiver has a very good performance since the number of BER is above $10^{-9}$ and $\mathrm{Q}$ factor is more than 6 . As mentioned, the height of the eye diagram defines the immunity of the received signal to the noise. The eye diagram for receiver 1 shows an eye opening of 0.00027 . For receiver 2 with $60 \mathrm{~km}$ fiber length, the received signal has BER of $1.918 \times 10^{-84}$. The Q factor of the received signal is 3.53 . Here, the BER analyzer shows a bad quality of signal. The eye opening of the eye diagram is 0.0000121 . It shows that the received signal in receiver 1 has more immunity to noise compared to the received signal in the receiver 1.

As shown in the example above, the result tells us that the BER is $1.10 * 10^{-10}$ and the Q factor is 6.30. There is an eye opening in the result of the example which is $2.585^{*} 10^{-5}$. The example is a quite good result since the BER is less than $10^{-9}$ and the $\mathrm{Q}$ factor is more than 6 . If all the receivers receive an output just like the example above, all objectives had been achieved.

Figure 9 shows the RF spectrum of I/Q components of the CO-OFDM WDM system for one user at the transmitter side. The RF power is measured about $(-10 \mathrm{dBm})$. Figure 10 shows the four OFDM spectrums after the WDM system. Four WDM channels starting from 193.05 THz to $193.2 \mathrm{THz}$ with channel spacing of $50 \mathrm{GHz}$, the power of the signals are about $(-44 \mathrm{dBm})$. Figure 11 shows the four OFDM signals after SMF with amplitude of $-28 \mathrm{dBm}$. The amplitude decreased because the noise and the dispersions of the fiber link. 


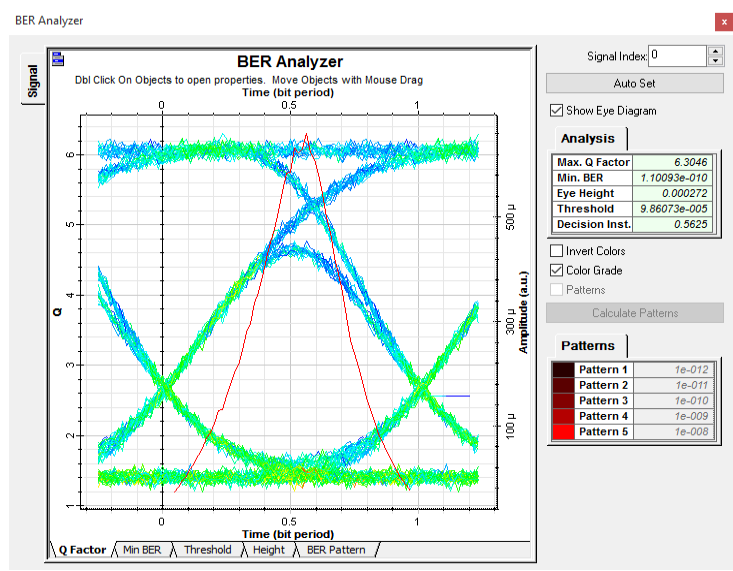

(a)

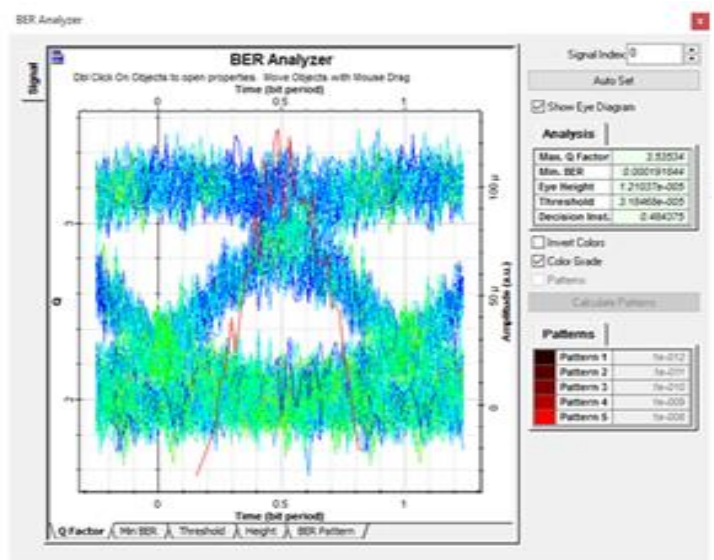

(b)

Figure 8. BER analyzer for receiver 1 with (a) $10 \mathrm{~km}$ and (b) $60 \mathrm{~km}$ fiber length
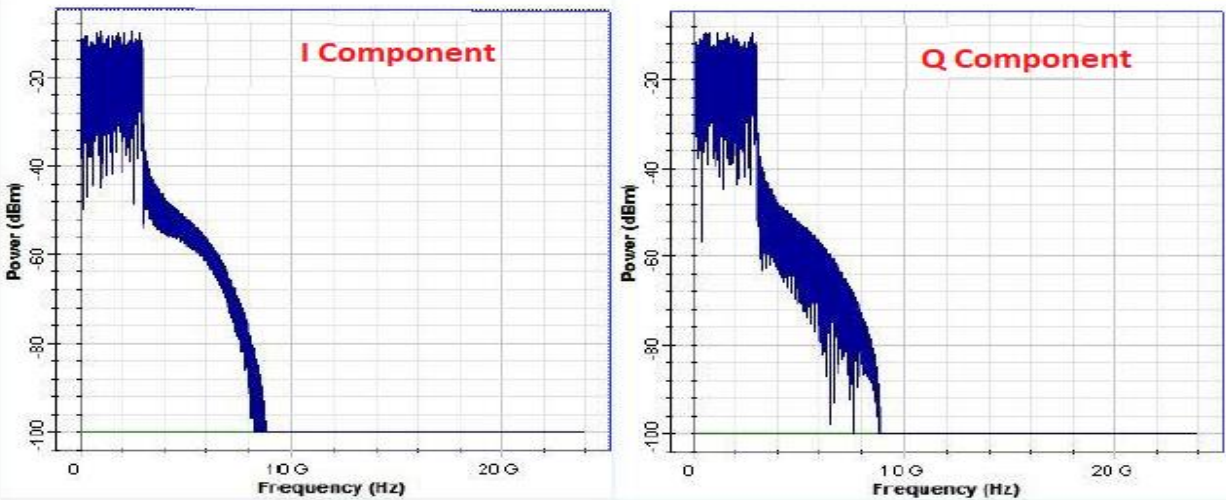

Figure 9. RF OFDM spectrum I/Q component at the CO-OFDM transmitter

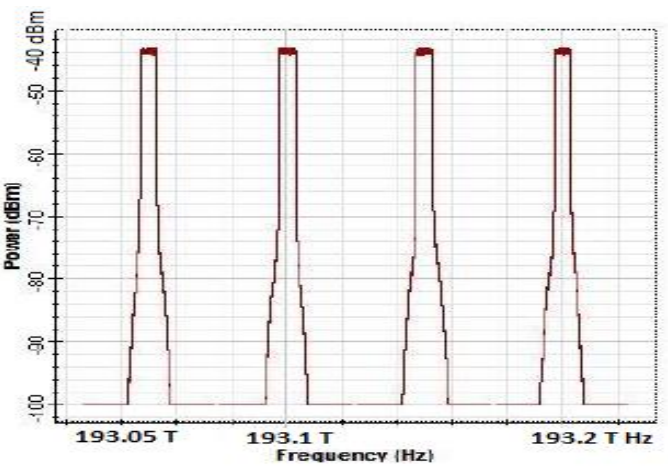

(a)

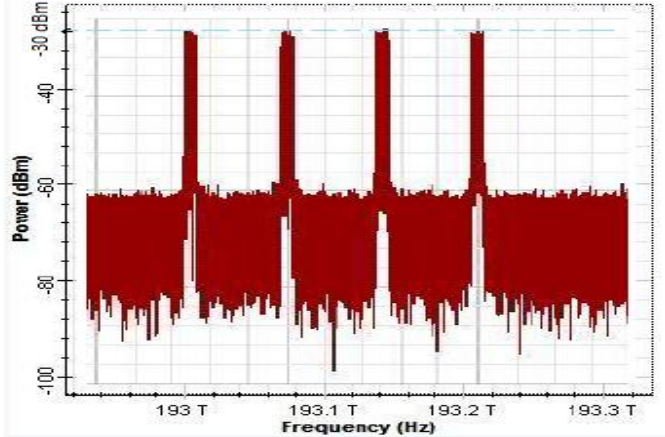

(b)

Figure 10. OFDM signal after WDM MUX (a) with 4 channels on the transmitter side and after SMF (b) at the receiver side

Figure 11 shows the constellation diagram of the WDM CO-OFDM system after $100 \mathrm{Km}$. As can be seen from the figure, the transmission is successful and the signal is easy to detect. It can be found out that the integration of CO-OFDM with WDM systems give a better performance of the system, in addition, that an easy for using the SMF with different wavelengths for increasing the data rates capacity to the total system. 


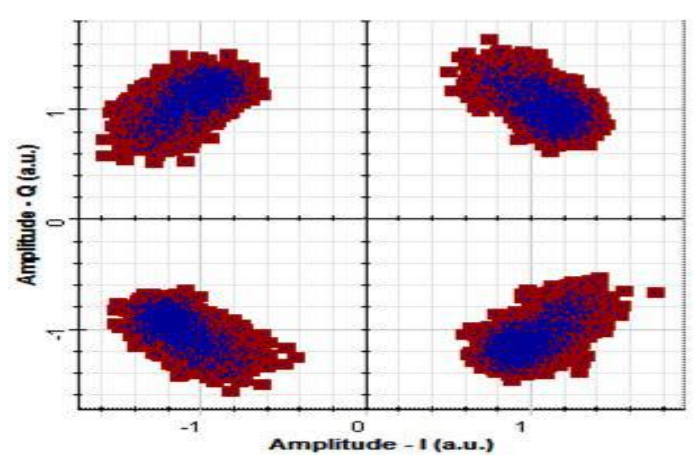

Figure 11. WDM CO-OFDM Constellation diagram for one user after $100 \mathrm{Km}$

\section{CONCLUSION}

The RoF system is essentially a transmission of analog signals as it distributes RF signals from CS to BS directly at radio carrier frequency. RoF offers low attenuation with broad bandwidth. It has the advantage of an immune system to the electromagnetic interference (EMI). A RoF system employing a WDM technique (WDM-RoF) is simulated using Optisystem 13.0. WDM can be considered the best method used in RoF. WDM enables transmission of multiple signals through a single fiber over large and can exploit the fiber network bandwidth. WDM enables the increasing of an optical fiber capacity compared to simple link of point to point which only enable to carry only single wavelength.

The performance of the integration of CO-OFDM system with a WDM system for long-haul transmission of $200 \mathrm{~km}$ with high data rate of $48 \mathrm{Gbps}$ was proposed, simulated, and analyzed. The WDM system consists of four channels to support the four OFDM bands with the channel space of $50 \mathrm{GHz}$. Each OFDM signal has a 12 Gbps bit rate which will provide $48 \mathrm{Gbps}$ overall data rate. The results show that the system is reliable and can provide significant high data rates with four wavelengths in one SMF fiber. Also, the results show that the noise and CD increases as the transmission distance increases.

\section{REFERENCES}

[1] B. U. Rindhe, et al., "Implementation of Optical OFDM Based System for Optical Networks," International Journal of Electrical and Computer Engineering (IJECE), vol. 4, pp. 767-781, Oct 2014.

[2] B. U. Rindhe, et al., "Implementation of Optical OFDM Based System for Optical Networks," Int. Conf. on Advances in Communication and Computing Technologies, 2014.

[3] H. Al-Raweshidy and S. Komaki, "Radio-over-Fiber Technologies for mobile communication networks, Link design for next generation wireless systems," J. Lightwave. Technology, vol. 28, pp. 136-138, 2010.

[4] A. H. Ali and A. D. Farhood, "Design and Performance Analysis of the WDM Schemes for Radio over Fiber System with Different Fiber Propagation Losses," Fibers, vol. 7, pp. 19, 2019.

[5] S. Singh, et al., "Performance Evaluation of Channel Estimation in OFDM System for Different QAM, and PSK Modulations," International Journal of Electrical and Computer Engineering (IJECE), vol. 1, pp. 140-150, Dec 2011.

[6] Mohan, "Full Duplex Transmission in RoF using WDM \& OADM Technology," IJERT, vol. 4, pp. 513-516, 2015.

[7] F. Nadeem, et al., "Performance Analysis of WDM-RoF System with CO-OFDM for Long Distance Communication," Int. J.l of Computer Applications, vol. 129, 2015.

[8] N. Praba and K. M. Ravikumar, "PAPR Reduction at Large Multi-User-MIMO-OFDM using Adaptive Data Detection Algorithm," Indonesian Journal of Electrical Engineering and Computer Science, vol. 12, pp. 10711080, Dec 2018.

[9] S. M. A. Satar, et al., "Coherent Optical - OFDM using 64QAM to high data rates $1.60 \mathrm{~Tb} / \mathrm{s}$ over $4500 \mathrm{~km}$," International Journal of Scientific and Research Publications, vol. 5, Sep 2015.

[10] K. Alatawi, et al., "Performance Study of 1 Tbits/s WDM Coherent Optical OFDM System," Optics and Photonics Journal, vol. 3, pp. 330-335, 2013.

[11] H. A. Mohammed and A. H. Ali, "Effect of some security mechanisms of the Qos VoIP application using OPNET," Int. J. Curr. Eng. Technol., vol. 3, pp. 1626-1630, 2013.

[12] A. A. Abdulrazzaq, et al., "QoS Performances Evaluation for Mobile WIMAX Networks based on OPNET," Int. J. Appl. Eng. Res., vol. 13, pp. 6545-6550, 2018.

[13] Y. Tang, "Coherent Optical OFDM Transmission Up to $1 \mathrm{~Tb} / \mathrm{s}$ per Channel," Journal of light wave technology, vol. 27, Aug 2009.

[14] S. Singh, et al., "Performance Evaluation of Channel Estimation in OFDM System for Different QAM and PSK Modulations," International Journal of Electrical and Computer Engineering (IJECE), vol. 1, pp. 140-150, 2011. 